\title{
The Application of Multi Materials in the Design of Ceramic Ware
}

\author{
Li Zeng \\ Hunan Arts and Crafts Vocational College, 413000, Yiyang, Hunan, China \\ 55249746@qq.com
}

Keywords: Ceramic Ware, Media Material, Multi Materials, Integration.

\begin{abstract}
People's needs, with the development of society, gradually tend to be diversified. Therefore, the selection and application of ceramic materials are in the continuous innovation, evolving from the entire ceramic texture of the original gradually into a comprehensive application of multi materials. This thesis analyzes the comprehensive and innovative application of multi materials in ceramic ware to discuss the integration and innovation of multi materials into the ceramic materials, which embodies the material aesthetics in the ceramic ware design. Furthermore, it investigates practical application in the ware design based on the modeling, functional and emotional design.
\end{abstract}

\section{Introduction}

The material is the carrier of the designer to express design intent, which contains the ingredient, the embodied property and texture. Moreover, it can be the expression of the media to the designer. It also can be referred as media material, which is the important manifestation in product design. The materials have different characters so that the various materials can bring people different physiological and psychological senses. Since ancient times, ceramic ware has been treated as the daily life appliances, taking the ceramic material as the main element. However, with the change of the times and people's lifestyle, ceramic ware design in the application of materials has become more innovative, which is properly illustrated by the multi material application.

\section{Ceramic and ceramic ware}

The ceramic material is the general term of pottery and porcelain. Ceramic is molded from the clay. With the bake of fire, it can be used by the people. Porcelain refers to be molded from porcelain clay with the high temperature sintering to be relatively sophisticated wares. Earthenware is simple and unadorned through the sintering of pure earth, making a person have a sense of simplicity by returning to nature and a quiet feeling of original state. Porcelain is formed from embryo as thin as cicada's wings together with smooth glaze, letting the ware with the noble and elegant temperament. The delicate lines highlight the exquisitely carved feeling, while the rich and colorful color of the glaze adds fashionable odor for fetal matter. Ceramic has distinct characteristics but without fixed shape. Consequently, the texture beauty can lead a person to endless aftertastes without losing pleasure.

Ceramic ware is the necessary instrument in people's lives. It has been thousands of years in using the ceramic material as unique expression language. Ceramic ware, in common life, includes tea set, tableware, wine set, coffee set, home furnishing, decoration apparatus etc. With the progress of science and technology and in-depth exploration of nature, a variety of new materials have emerged. Designers will try to apply the multi materials to the ceramic ware design in order to seek the new forms of language to increase the diversity. Through the direct relationship between specifically practical ware and the user, ceramic ware design not only needs to correctly reflect the texture beauty of different materials in the design, but also needs to study the emotional embodiment of the texture of the material to make multi materials integrated in the design of ware. 


\section{The integration of multi materials in ceramic ware}

The material is the foundation of substance in the design. Each kind of material has its own characteristics. The integration of different materials conveys diverse formal languages and emotional expressions. The multi material application, in the ceramic ware design, becomes the tendency of modern design. The previous ceramic ware simply refers to the products of ceramic material. With the development of society, people's living standard is constantly improved, which makes a lot of products not merely depend on a single material to complete their appearance design, the use and other function designs. Ceramic ware begins to further carry people's aesthetic taste, the pursuit of fashion and life attitude. The relationship between ceramic ware and the user is not only reflected in the use process, but also shortens the distance between people and objects when their relationship emerges at the same time. Therefore, people are no longer satisfied with basic daily use of ceramic ware. Moreover, they raise their need into the spirit level. In the wake of the development of the contemporary ceramic ware, designers boldly pursue the performance of the individual in the creation, breaking the traditional recognition of a single material in the ceramic ware to people and interpreting the new artistic language of ceramic ware combined with multi materials.

Bamboo and wood. Innovation and application of bamboo and wood in the ceramic product design optimizes utility function and circulation method of the ceramic product, avoids the defects of ordinary ceramic materials, and elaborates the functionality of ceramic products. Bamboo and wood have the advantages of toughness, breakage-proof and good heat insulation, which allows small deformation. To some extent, it makes up defects of the ceramic firing in high temperature, such as uncertainty and difficulty in control.

The use of bamboo and wood in ceramic ware has a long history. Generally, it is shaped in the pot handle, hoop handle and tray. Qiao Ano said: "the wood often makes itself turn into the performance of life, conflict, and noise, it has a meticulous soft feeling and fresh wood flavor, which makes you have the feeling of constant close. It seems that it has a strong affinity". There is a large selection for bamboo, which has great consideration in the choice of delicate and exquisite wood and the pattern itself. Bamboo material is restricted to the single material and only one subtraction in the production process. In the combination of the ceramic ware, it can take the skills of riveting, tenoning, winding or stitching. In the modern ceramic ware design (Figure 1 and Figure 2), it embeds the art processing of bamboo material. Bamboo material can bring a person a kind of warm in the psychological effect and the simple but elegant porcelaneous beauty in vivid reflection. Moreover, it is the demand of artists to pottery and porcelain in the whole expression of emotion, concept and visual art, which is the necessary choice under the influence of contemporary art.

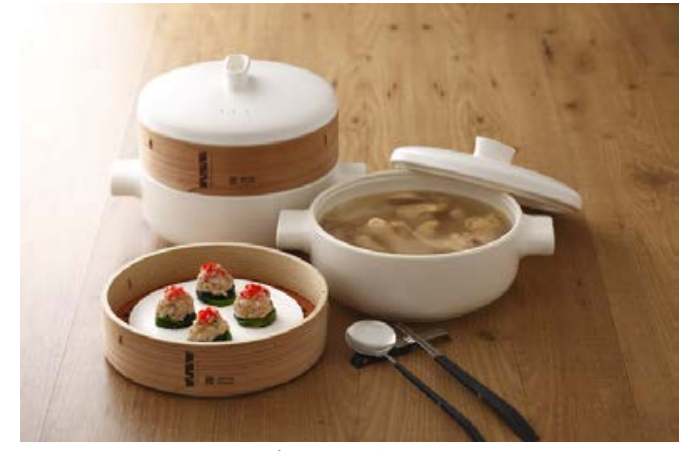

Figure 1

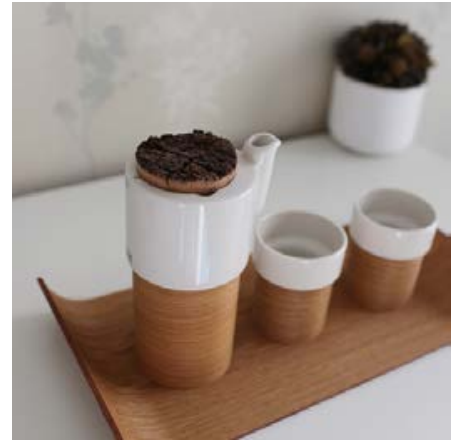

Figure 2

Metal. Metal has the functions of glossiness, ductility, electric conduction and heat transmission. The development and application of metal is inseparable from the progress of the times and improvement of science and technology, which plays an irreplaceable role in the actual application. The glossiness and texture patterns of the metallic materials form the emotional factors of infectivity and modern sense. For example: copper material illustrates simple, unsophisticated, far-reaching, and solid feeling and unique beauty to people. Stainless steel is naturally silver gray with delicate and bright luster and high reflection, which gives a person a sense of thorough feeling. In the Figure 3, the 
designer uses stainless steel combined with ceramic material; it can compensate uneasy display of ceramic material from the aspect of modeling. Meanwhile, the contrast formed between the two materials in the surface texture can produce a strong visual effect.

Glass. The glass is melted and formed by quartz, feldspar, dolomite and other raw materials. Its main features include high transparency and glossiness compared with other material. Some of the characteristics of glass are similar to the ceramic, which can show elegant and transparent form of art. It is more transparent than the ceramic. The effect of light penetration makes color more abundant. Therefore, the aesthetic value of glass materials is also gained identity by people.

There are two forms of the combination of the glass with ceramic wares. One is the glass as the auxiliary decoration materials, the other one is the glass as enamel material directly into the ceramic surface. Figure 4 is a set of ware by a Japanese designer Tanaka Misa, which is formed in the combination of firing white ware material and translucent glass by the sticky method, showing a kind of quiet beauty. Ware is elegant and concise. The pedestal of glass serves as a foil to the mild ceramics, drifting between the translucence and full transparency. Two kinds of materials are naturally fused, which gives people visual sense in the new outlook.

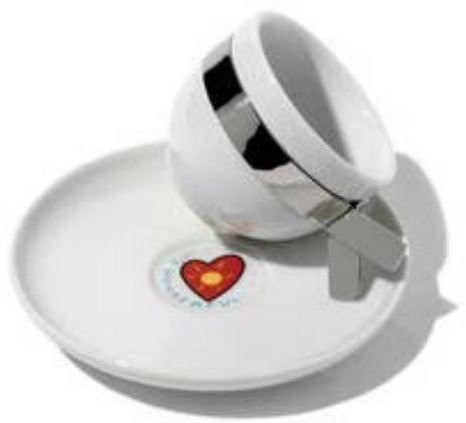

Figure 3

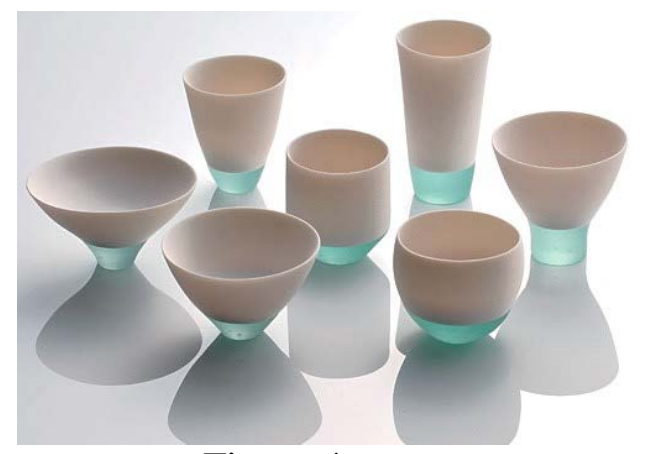

Figure 4

\section{The design of ceramic ware and the application of multi material}

The design of ceramic in ware bears medium of art thought as well as reflects the ware itself charm. If we can skillfully utilize the combination of ceramic material itself and other multi material, we will not only make comparisons between texture and arrangement, but also make up the limitation of material itself on the foundation of exerting ceramic characteristics. The characteristics of ware design are more than perfect performance of modelling but respect the material nature. The material in the ceramic ware design is the material basis of product. Apart from the functional performance of material itself, it has its unique material characteristics and emotion, which can reflect the beauty of different materials. Designers constantly broaden their horizons, try the application of new materials, scientifically and reasonably use the characteristics of media materials, and combine the shape and function of the ware itself with the expression of emotion, to broaden the design ideas, which will bring new atmosphere to the design for ceramic ware and further interpret today's artistic view.

The combination of functional design and material. The functional design itself is based on the principle of practicality, Mr. Zhang Dainian pointed out, "the mode of thinking, in the dominant position in Chinese traditional philosophy, features with the emphasis of harmony, overall, and practicality." Obviously, any practical design treats functionality as the design concept, so is the design of ceramic ware. In the process of ware design, designers need to consider many factors. Based on the function, how to further perfect the functionality of ceramic ware with the proper use of the multi materials is the key of design task. If we want to realize the ceramic ware function, we must rely on the media material, which needs to make each kind of material show its special properties in use. The application of multi materials in the functional design should consider the rationality of function and the convenience of use.

With the foundation of functional design, designers need to exert use function by the scientific processing technology and the structural style of the product. When users use this product, they will generate a sense of trust and security for the product. Figure 5 is the design of a double mouth tea set. 
Using the bamboo plate to produce the holder can have the possibility of realizing double pouring, which perfects and embodies the design idea of the designer on the function realization. Innovative application of multi materials improves the practical performance of the ware in a great extent, and avoids the defect of common ceramic material, which makes the practical function of ceramic ware more perfect. Therefore, it not only strengthens function realization of the ceramic itself, but also enriches the design space of ceramic ware.

The combination of appearance design and material. It is one of the most important links that the application of multi materials should be unified with thought by the designers in the ware design process. Sometimes, the particularity of media material will broaden the works and stimulate thinking space of the user. Products of different design categories need to be performed by multi materials. However, the multi material properties and its functions will naturally influence or control the content and form of it. Modern design of ware is more than to meet the single material. At the same time, the combination of multi materials is the media in the process of ware design by the designer. It is equally important in the consideration of material application and techniques processing of appearance to a piece of work. In the design of modern ceramic ware, the intervention and use of other materials can bring and subvert people's visual bias. The key lies on whether the material itself can bring the impulsive, passionate and intelligent thoughts. Figure 6 is the design of a set of ceramic ware with the prototype of chemical instrument. It combines the unexpected metal of the ceramic with wood. The sense of form is so creative that can attract the eyeballs of consumers. Therefore, the ceramic ware must be designed according to different characteristics of materials with the consideration of the organic unity between the texture and color of the material with practical function.

The multi materials are used in the ceramic ware, which can have perfect combination with the main materials - ceramic. The appearance skills are embedded with implant, superposition, surrounding and etc.. The modelling expert Roland pointed out: "many changes of man-made objects are consistently associated with material and its manufacturing method". When the material is changed, it will bring the changed morphology. If the processing method of molding is varied, it will lead to the changed morphology. That is to say, the diversification of multi materials' function makes the appearance of ceramic ware achieve diversified characteristics. Ware modelling requires the designer to give full consideration to the related physical characteristics of multi materials, furthermore, he/ she needs to consider whether the processing properties of materials can meet user's requirements to gain durable, practical, beautiful and elegant style.

The combination of emotional design and materials. Visual beauty tends to stimulate the user to produce diverse mentally emotional reactions. The emotion is the core of design. Moreover, the injection of emotions in the design is the ultimate problem to be solved in the design field. Designers not only need to understand the different physical characteristics of multi materials, but also need to experience emotional attributes and perceptual properties included in of materials. In this way, they can translate them by processing into emotional objects and endow the products a profound artistic connotation, emotion and human experience. In the Figure 7, the ware composes of the wood branch type handle and ceramic material, which gives people the feeling of returning to nature and the expression of emotion of closing to the nature. That is to say, the good ceramic ware design is more than the practical functional design, but it should have the affinity of modeling as well as a rich emotional experience.

The psychology of design believes that design directly resorts to human's emotion experience, which regards the aesthetic feeling as the core of the experience. The artistic characteristics of ceramic are generated from the visual experience in the use. Whether ceramic ware can make people obtain the aesthetic feeling of emotion experience depends on whether its objective aesthetic characteristics are fully possessed in the first place. Most important, it also relies on the user's psychological feedback activities, which is indispensable, necessary and sufficient condition to the aesthetic feeling. Materials rising from the material media to mental performance are the starting point of multi materials in the artistic expression. The abstract feeling of material plays the decisive role in it. Different materials have different emotional features, and diverse textures give different psychological guidance. The deep understanding and acquaintance of the material emotion by the 
designer can directly affect the application of materials in the design of the ware. For example, compared with lightsome and transparent glass, the rough and unsophisticated ceramic is relatively thicker and solider since the glass have the light transmittance and smooth surface which looks bright and airy. The natural and modesty wood significantly differs from the simple and natural metal material since the use of bamboo material possesses with more warm feeling of the human feelings, while the metal has fashionable and technical texture.

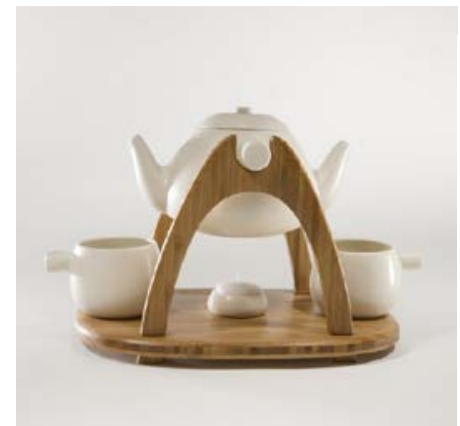

Figure 5

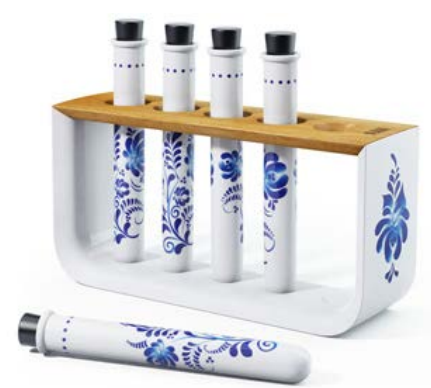

Figure 6

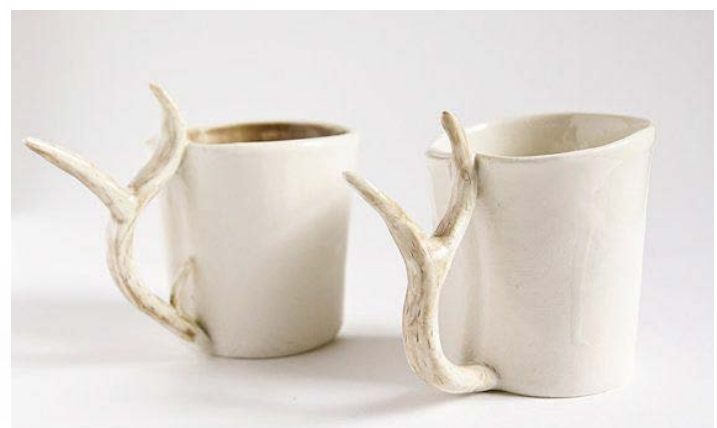

Figure 7

\section{Conclusions}

The application of multi materials in the ceramic ware design provides more patterns to express thoughts, feelings, and ideas for ceramic creators. Moreover, it mobilizes the inspiration and creativity and expands the scope and the development of ceramic ware design. As for the selection and application of materials, we should fully retain the multiple personality, of which the most important is to explore the internal rules of the material and master the performance differences. Faced with multi materials to design the ceramic ware, designers should think of its physical and chemical characteristics as well as understand emotional experience brought to people. In this way, they can transfer the lifeless material into vivifying products and endow ceramic ware design more profound emotional and artistic connotation.

\section{References}

[1] Yalin Zhang, Shouhan Liu. The research on the domestic ceramic design with the comprehensive materials[J]. The ceramic industry in China, (2009.3)

[2] Liping Chen . Discussion about the application of materials in the ceramic sculpture creation[J]. The seminar thesis of the development trend of ceramic art design in China, (2006.6)

[3] Chenchen Du. A variety of materials in the design of modem tea emotional experience evolving research[D]. Wuhan University of Technology, (2013.5)

[4] Qian Dong . The application research of household porcelain combined with the multi materials[J]. Popular literature, (2014.12)

[5] Mingyu Ceng, Hanxin Zhou . The application idea of the multi materials in the domestic ceramic design [J]. China's ceramics, (2008.10)

[6] Fanqian Meng. The analysis of the innovative application of the wooden materials in the design of ceramic products[J]. Design, (2013.3)

[7] Chengen Jiao. The aesthetic research on traditional glass equipment qualitative [D]. Hunan Normal University, (2008.5)

[8] Yong Yu,Yalin Zhang. The analysis of daily-use ceramics material quality embodiment [J].China ceramic industry, (2002.10) 